\title{
Optic neuropathy due to allergic fungal rhinosinusitis
}

\author{
Jiji Tresa Cyriac, Tambi Cherian', Wasna Ali Hadi², Joyce Jose ${ }^{2}$ \\ Departments of Ophthalmology, ${ }^{1} E N T,{ }^{2}$ Pathology, Gulf Medical College Hospital and Research Centre, Gulf Medical University, Ajman, UAE
}

\section{ABSTRACT}

An uncommon case of allergic fungal rhinosinusitis presented to the ophthalmology outpatient department of our hospital with complaints of blurred vision in the right eye of a few days duration and vague complaints of pain around the eyes. The visual acuity on examination was grossly reduced in the right eye and normal in the left eye. Color vision was normal. Anterior segment examination including pupils was normal. Dilated fundus examination was normal except for temporal pallor in the right optic disc. Automated perimetry and magnetic resonance imaging (MRI) scan of brain and orbit were done. The imaging report showed a bilateral pansinusitis with pressure on the right optic nerve. Perimetry showed a superior field defect on the right side. ENT consultation and computed tomography (CT) with contrast helped to diagnose this as a case of allergic fungal rhinosinusitis. The patient was started on systemic steroids under the care of the ENT surgeon. After a few days, pre-operative assessment showed a gross improvement of visual acuity. Endoscopic sinus surgery was done to remove the polyps and thick mucus material. Histopathologic examination confirmed allergic fungal mucin. Days after surgery, the visual acuity improved further and repeat perimetry showed gross improvement in the visual field. Good history taking and a detailed ophthalmic examination, keeping in mind the probable causes of loss of vision of few days duration with no findings other than a decreased visual acuity and a suspicious disc, were key to the early diagnosis and investigation in this case. This helped in early referral and management of the case before permanent damage and irreversible visual loss occurred. The optic nerve is a cranial nerve which, once damaged permanently, will not regenerate. The amount of sinus involvement was extensive on both sides and invariably the left optic nerve would have been involved in a few days, if intervention was delayed.

Key words: Allergic fungal rhinosinusitis, optic disc, optic neuropathy, perimetry, visual loss

\section{Introduction}

Early cases of optic neuropathy are difficult to diagnose because the typical signs may not be present. The symptoms develop gradually as further damage to the nerve occurs. High level of clinical suspicion is required in all cases of acute loss of vision with normal anterior segment. Allergic fungal rhinosinusitis is an uncommon cause of optic neuropathy. ${ }^{[1]}$

\section{Case Report}

A 22-year-old, healthy Asian male presented to the

\begin{tabular}{|l|l|}
\hline \multicolumn{2}{|c|}{ Access this article online } \\
\hline Quick Response Code: & Website: \\
\hline & www.ruralneuropractice.com \\
\cline { 2 - 3 } & \\
\hline
\end{tabular}

Ophthalmology Outpatient Department with the complaints of blurred vision in the right eye of 3 days duration and feeling of heaviness and pain around the eyes for the past 10 days. There was no history of trauma or drug intake. He was a non-alcoholic and nonsmoker, with no other relevant history. On examination, visual acuity was 3/60 in the right eye and 6/6 in the left eye, color vision was normal, pupils were equal and reacting to light. No (relative afferent papillary defect). Extraocular muscle movements (EOM) were full in both eyes. Anterior segment of both eyes showed no abnormality. Fundus (dilated with $1 \%$ tropicamide) was normal in both eyes except for temporal pallor of optic disc RE The patient was advised perimetry and magnetic resonance imaging (MRI) brain and orbit to identify the cause of pressure on the right optic nerve. An automated perimetry was done which showed a superior field defect in the right eye MRI showed pansinusitis of both sides with onodi cells on the right side, with slight impression on the right optic nerve in the optic canal. He was advised ENT consultation. Comupted tomography $(\mathrm{CT})$ with contrast was done Figure 1. A

Address for correspondence:

Dr. Jiji Tresa Cyriac, Department of Ophthalmology, Gulf Medical College Hospital and Research Centre, Gulf Medical University, Ajman, UAE. E-mail: jjtresa@yahoo.co.uk 


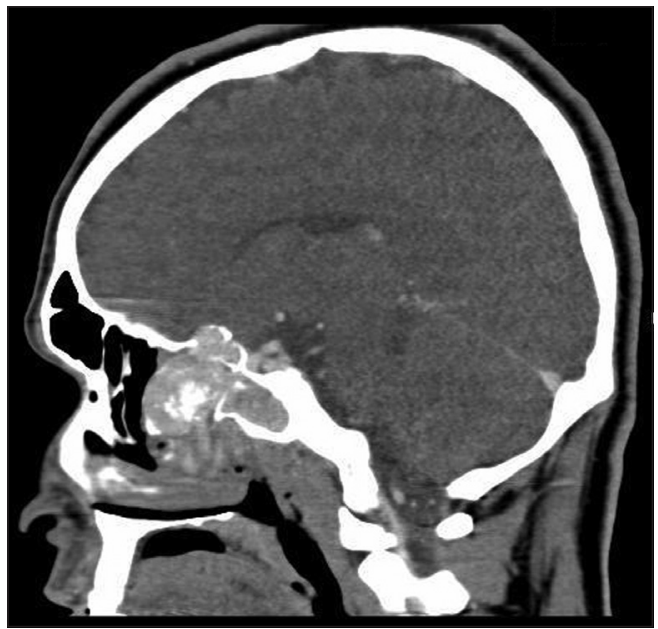

Figure 1: Sagittal CT contrast enhanced

diagnosis of allergic fungal rhinosinusitis was made based on clinical and radiological findings. The enlarged and inflamed sinuses, especially the ethmoid sinus, were causing pressure on the optic nerve, mainly in the region of the optic canal (pressure due to inflammatory edema). The patient was started on systemic steroids after baseline investigations by the ENT surgeon and surgery was planned. Preoperative follow-up after one week showed gross improvement in visual acuity, $6 / 12 p$ $\mathrm{RE}, 6 / 6 \mathrm{LE}$, color desaturation in the RE, pupils were normal, and fundus showed temporal pallor of disc RE. Surgical removal of the polyps, opening the sinuses and removal of the allergic mucin, permitting ventilation was done. Sample was sent for histopathology and special staining to confirm the diagnosis. Microscopic examination showed large pool of eosinophilic mucin with numerous Charcot-Leyden crystals. Grocott's methenamine silver staining showed broad, septate, branching hyphae consistent with Aspergillus [Figure 2]. Follow-up after one month showed visual acuity $6 / 10$ $\mathrm{RE}, 6 / 6 \mathrm{LE}$ and fundus picture was the same. A significant improvement in the perimetry findings suggested that once the cause of compression on the right optic nerve was treated, the function improved, and early treatment prevented permanent damage. Comparison of the perimetry findings in the right eye, there was significant improvement after systemic steroids and the sinus surgery. Regular follow-up in the ENT and Ophthalmology clinics was advised as this condition tends to be recurrent.

\section{Discussion}

The optic nerve contains retinal ganglion cell axons that extend posteriorly from the globe, through the orbit and optic canal to reach the optic chiasm. An injury

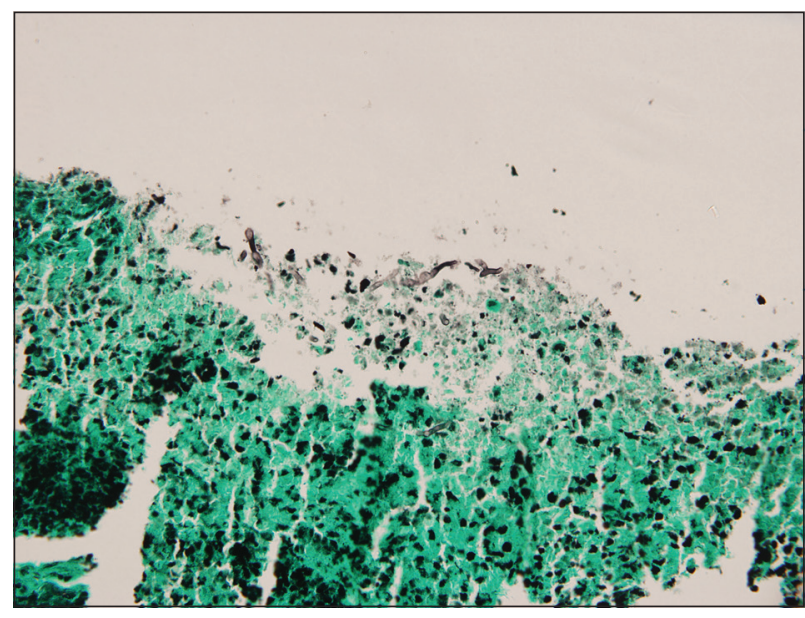

Figure 2: Grocott's methenamine silver staining showing branching hyphae

to the optic nerve anywhere along this pathway by an extrinsic lesion is termed compressive optic neuropathy. It is most vulnerable to injury where it is adjacent to bone or in a confined space like the orbital apex or optic canal. The clinical hallmark of compressive optic neuropathy is progressive vision loss. Other features include dyschromatopsia, RAPD, field defects, and optic atrophy. A delay in the diagnosis is not uncommon since the vision loss is insidious and the clinical findings may be missed. Clinicians should consider compressive optic neuropathy in the differential diagnosis of unexplained, asymmetric vision or visual symptoms that are unresponsive to corticosteroids. ${ }^{[1]}$ Optic nerve compression by an extrinsic lesion has been postulated to cause atrophy of ganglion cell axons through either ischemia or mechanical disruption of axonal transport. ${ }^{[2]}$ Depending on the location of the lesion, proptosis, squint, eyelid malposition, anosmia, facial numbness, or pituitary dysfunction may occur. Whenever there is clinical suspicion of compressive optic neuropathy, a neuroimaging study is mandatory. With the sensitivity and specificity of modern neuroimaging, a negative scan essentially rules out the possibility of compression as the cause of vision loss. ${ }^{[1]}$ MRI is the imaging modality of choice because of the excellent soft-tissue resolution of the anterior visual pathway and parasellar area. For the sinus problem, CT with contrast is more appropriate. Management of compressive optic neuropathy is sometimes difficult, given the proximity of compressive lesions to critical neurovascular structures in the orbit and intracranial space. In this case, management of allergic fungal rhinosinusitis was important to relieve the pressure on the optic nerve. ${ }^{[3]}$ Fungal rhinosinusitis is a cause of chronic rhinosinusitis. ${ }^{[4]}$ It comprises $5-10 \%$ of all chronic sinusitis and is seen in areas of high humidity. ${ }^{[4]}$ Patients diagnosed with Allergic fungal rhinosinusitishave a history of allergic rhinitis. Thick 
tenacious and viscous fungal debris and mucin are the characteristics of this condition and allergic mucin is pathognomonic of AFRS. Its color may be brown to dark green. Inflammatory reaction causes tissue edema, obstruction of ostia and stasis within the sinuses. This creates an ideal environment for proliferation of the fungus. This leads to the activation of cytokines, causing local infiltration of eosinophils, mast cells and increase in IgE production. Mast cells release histamine causing local inflammatory changes. Radiologic features are classical. MRI features: High protein and low water concentration of allergic fungal mucin coupled with high water content within the surrounding edematous sinus mucosa gives rise to specific MRI characteristics. ${ }^{[5]}$

\section{Conclusions}

Good history taking and thorough ophthalmic examination were key to the early diagnosis and timely management of this case before it caused a major permanent damage to the right optic nerve and well before it started pressing on to the left optic nerve. Though such cases are not very common, subtle changes on fundus examination should give rise to clinical suspicion and imaging studies should be done urgently. Prompt treatment reduced the pressure effects on the optic nerve. Hence, vision and field changes grossly improved.

\section{References}

1. Attallah M, Hashash M, al-Muhaimeed H, Dousary S, al Rabah A, Kharashi S. Reversible neuropraxic visual loss induced by allergic Aspergillus flavus sinomycosis. Am J Rhinol. 1999;13:295-8.

2. e-medicine [Online]. Optic Neuropath: Compressive, 2010. Available from: http://emedicine.medscape.com/article/1217005-overview. [Last cited on 2010 Oct 16].

3. Marple BF, Gibbs SR, Newcomer MT, Mabry RL. Allergic Fungal Sinusitis-Induced Visual Loss. Am J Rhinol 1999;13:191-5.

4. e-medicine [Online]. Optic Neuropath: Compressive 2009. Available from URL: http://emedicine.medscape.com/article/834401-overview. [Last cited on 2010 Oct 16].

5. Reddy CE, Gupta AK, Singh P, Mann SB. Imaging of granulomatous and chronic invasive fungal sinusitis: Comparison with allergic fungal sinusitis. Otolaryngol Head Neck Surg 2010;143:294-300.

How to cite this article: Cyriac JT, Cherian T, Hadi WA, Jose J. Optic neuropathy due to allergic fungal rhinosinusitis. J Neurosci Rural Pract 2011;2:180-2.

Source of Support: Nil. Conflict of Interest: None declared. 